\title{
Simple Iterative Channel Coding and Modulation for Harbor Wireless Communications
}

\author{
Olyvia Fransiska Andriani ${ }^{\mathrm{a}, \mathrm{b}, 1}$, Khoirul Anwar ${ }^{\mathrm{a}, \mathrm{b}, 2}$, Nachwan Mufti Adriansyaha, \\ ${ }^{a}$ The University Center of Excellence for Advanced Intelligent Communications (AICOMS), \\ ${ }^{b}$ School of Electrical Engineering, Telkom University, Bandung, 40257, Indonesia \\ Corresponding author: ${ }^{1}$ olyviafransiska@student.telkomuniversity.ac.id, ${ }^{2}$ anwarkhoirul@telkomuniversity.ac.id
}

\begin{abstract}
Monitoring systems at the harbor require a simple communication technique to save battery energy, strong in error correction, and suitable to the harbor channel model, of which the channel is predicted to have more number of path. This paper proposes (i) a harbor channel model and (ii) a simple iterative repetition coding for harbor wireless communication of monitoring systems. The harbor channel model is derived using a series of computer simulations based on real-field harbor environmental parameters, while the proposed iterative channel coding is based on the repetition codes assisted by a doped accumulator (ACC) with a doping technique. Channel coding and doping rates are designed using an Extrinsic Information Transfer (EXIT) chart and are evaluated using a series of computer simulations in terms of Bit Error Rate (BER) and Frame Error Rate (FER) performances with an outage performance as the theoretical baseline for comparison. Since the EXIT curve of the repetition codes has steep curve, we use a non-Gray Quadrature Phase Shift Keying (QPSK) to achieve a better matching between the decoder and the demapper of harbor channels. We found that the proposed simple repetition codes and non-Gray demapper provide excellent performances for harbor communications with a cliff of BER curve. The results of this paper are expected to contribute to the development of a reliable wireless communications for the harbor monitoring applications.
\end{abstract}

Keywords- Harbor wireless communication; container monitoring; channel model; iterative decoding; repetition codes; doped accumulator; non-Gray modulation.

Manuscript received Apr. 6, 2020; revised May. 18, 2020; accepted Jun. 10, 2020. Date of publication Feb. $28,2021$. IJASEIT is licensed under a Creative Commons Attribution-Share Alike 4.0 International License.

\section{INTRODUCTION}

The need for delivery via sea freight is increasing, where the 2018 annual report of PT Pelabuhan Indonesia II shows that container flow reached 7.6 million twenty-foot equivalent units (TEUs), which is $7.57 \%$ higher than the Corporate Budget Plan (RKAP) in 2018, increased about $10.33 \%$ from the container flow in 2017 [1]. Along with the increase of container flow, the monitoring process is significant to the improvement of container logistic service at the harbor. It is also motivated by the Industrial Revolution 4.0 development of automation and digitalization. This condition is on the right track towards a smart harbor and smart supply chain of the $4^{t h}$ generation harbor, where every process in the harbor is monitored in a single integrated system [2].

One of problems of reaching a level of smart harbor is unoptimized communication systems of the harbor to provide excellent services. The harbor communication systems should be designed carefully according to the harbor environmental characteristics.

A harbor in general has a lot of metal obstacles causing the transmitted signal experiences reflection, diffraction, and scattering. No special treatment to this phenomenon may cause worse communication performances. The signal from a different path having a different phase make severe intersymbol interference (ISI) that corrupt the received signal called as the multipath fading effect [3]. Consequently, the harbor communication systems need a channel coding 


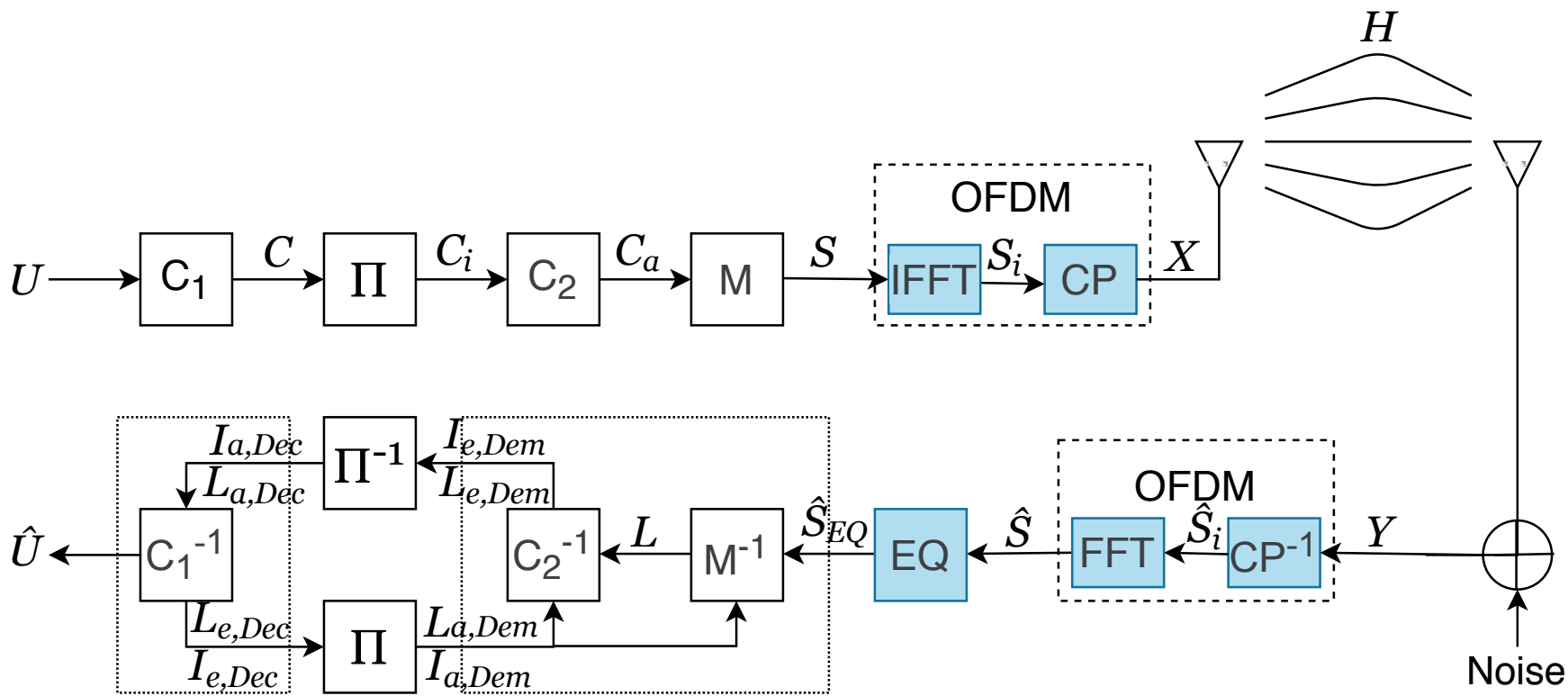

Fig. 1 The transmitter and receiver structure of the proposed iterative channel coding for harbor wireless communications.

that is suitable for the harbor environment to avoid power inefficiency.

Some studies on channel model have been conducted, for example by [4], [5], [6] and [7], proposing channel models of several communication system using a New York University Wireless Simulator (NYUSIM). This paper proposes harbor channel model based on the methods used in [4], [5], [6] and [7]. For channel coding, research in [8] and [9] have proposed Bit-Interleaved Coded Modulation with Iterative Detection/Decoding (BICM ID) using repetition codes and extended mapping. Performance improvements have been obtained with a method in [8] and [9] using an added doped accumulator (ACC) as proposed by [10].

This paper first proposes a harbor channel model and then proposes a reliable channel coding for wireless communication systems for the harbor communications. Channel model is needed to be investigated such that the characteristic and theoretical performance, for example in terms of outage performance, can be obtained [6]. We consider Tanjung Priok Harbor in North Jakarta as an example of harbor representing the characteristics of general harbor, especially in Asia, having international connectivities. Tanjung Priok is also the largest and busiest harbor in Indonesia [11].

The proposed channel model in this paper is a small scale fading channel model in the form of a Power Delay Profile (PDP) representing the power and the delay taken from thousand samples of simulations [6]. We further propose a channel coding using simple repetition codes with iterative decoding helped by a non-Gray demapper with doped ACC, where the design is optimized using extrinsic information transfer (EXIT) chart under the additive white Gaussian noise (AWGN) channel and the proposed harbor channel model.

Our contributions are summarized as follows:

1) Channel model for harbor wireless communications is proposed based on real-field environmental parameters. Indonesian harbor is taken as an example.

2) Outage performances of harbor channel model with coding rates $R=\{1,1 / 3,1 / 9\}$ are derived based on simulations as the theoretical references for harbor wireless communications.

3) Optimal Iterative decoding-based repetition codes are proposed to be one of the strong codes after a combination with non-Gray QPSK with doped ACC.

4) Optimal soft demapper of non-Gray QPSK is presented, where a distance parameter $d_{p}$ is optimized to avoid infinite value during practical computation.

This paper is organized as follows. Materials and method are presented in Section II. Results and discussion is presented in Section III. Finally, some concluding remarks are presented in Section IV.

\section{Materials And Method}

\section{A. System Model}

Fig. 1 shows a structure of the transmitter and the receiver of the proposed harbor wireless communication systems, where detailed Tanner graph is used to illustrate the doping technique. Orthogonal frequency division multiplexing (OFDM) is applied to mitigate the inter-symbol inferference (ISI) after the modulator $\mathrm{M}$. At the transmitter, information is eccoded using repetition codes with coding rate $R=1 / 3$ and $R=1 / 9$.

The encoded bits are interleaved by $\Pi$ and and further encoded by doped ACC. The ACC-encoded bits are then mapped with non-Gray QPSK modulation in block $M$. OFDM block consists of the Inverse Fast Fourier Transform (IFFT) and Cyclic Prefix (CP) is then introduced to add the $\mathrm{CP}$ to avoid the intersymbol interference (ISI) in the multipath fading channels. 


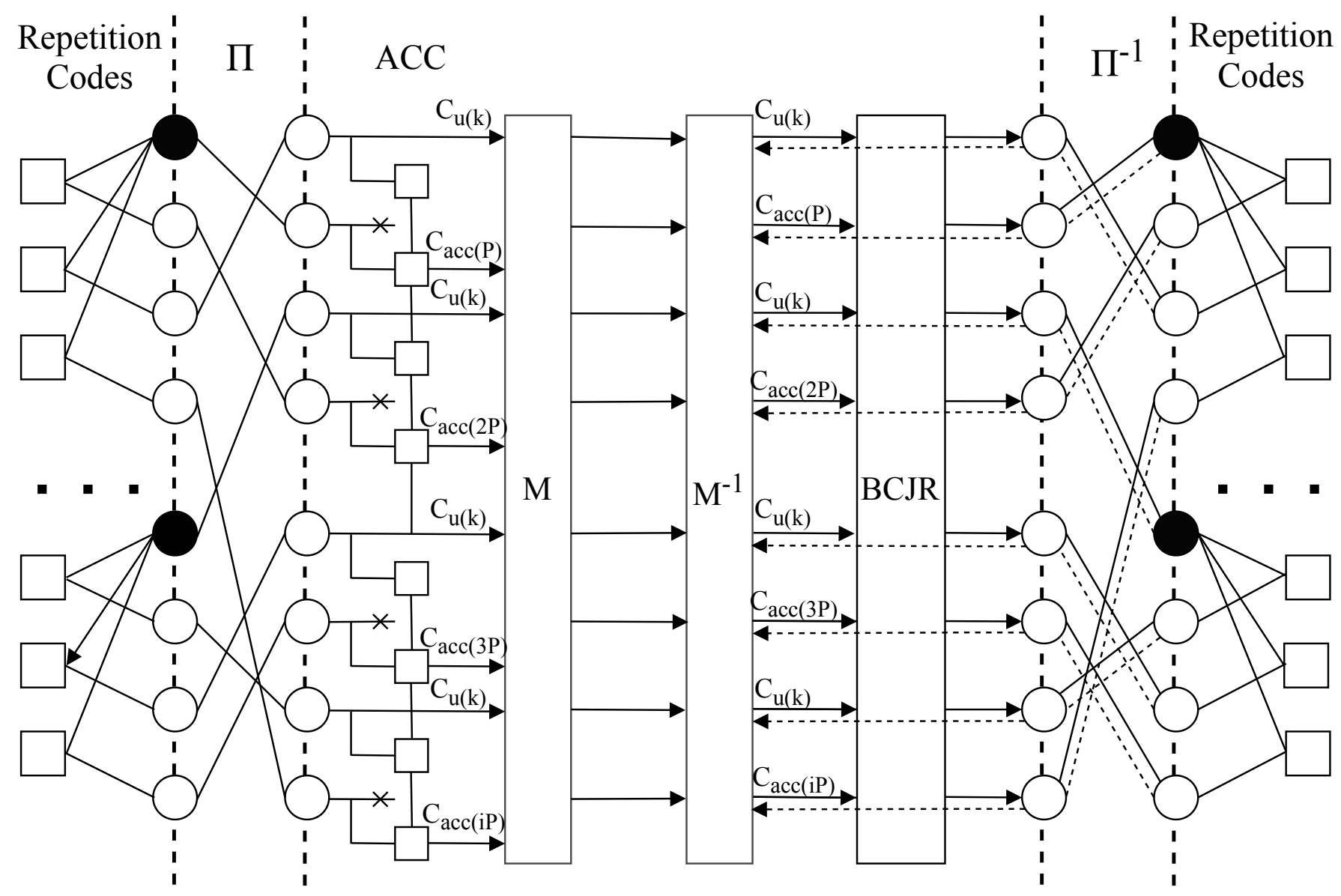

Fig. 2 Detailed Tanner graph between transmitter and receiver of the proposed iterative channel coding with doped accumulator.

The signal is transmitted through AWGN and multipath Rayleigh fading channels. The received signal can be expressed as

$$
\mathbf{y}=\mathbf{H} \cdot \mathbf{x}+\mathbf{n},
$$

where $\mathbf{H}$ is the Toeplitz channel matrix and $\mathbf{x}$ is the vector of symbols and $\mathbf{n}$ is the noise vector following Gaussian distribution of $\mathcal{N}\left(0, \sigma^{2}\right)$ with zero mean and variance of $\sigma^{2}$. In AWGN channel, the matrix $\mathbf{H}$ is an indentity matrix $\mathbf{H}=\mathbf{I}$, while in the block Rayleigh fading channels, the matrix $\mathbf{H}$ is an identity matrix multiplied by channel gains following Rayleigh distribution. The probability density function of the received signals under the block Rayleigh fading channels is expressed as

$$
P(y)=\frac{y}{\Gamma} \exp \left(-\frac{y^{2}}{2 \Gamma}\right),
$$

where $\Gamma=E\left[y_{I}^{2}\right]=E\left[y_{Q}^{2}\right]$ is the expected average power of the received signals with $y_{I}$ and $y_{Q}$ being the real and imaginary parts of the received signals $y$, respectively.

At the receiver, $\mathrm{CP}$ is removed at the block of $C P^{-1}$ and is further processed by the FFT block. The result of OFDM block is equalized using Minimum Mean Squared Error (MMSE) Equalizer on $E Q$ block. MMSE-equalized signal can be expressed as

$$
\mathbf{y}_{e q}=\frac{h^{*}}{h \cdot h^{*}+\sigma^{2}} \cdot \mathbf{y}
$$

with $h^{*}$ being the complex-conjugate of $h$. The signal $y_{e q}$ is further demapped by $M^{-1}$ to the log-likelihood ratio (LLR) domain. LLR is then decoded using BCJR in $C_{2}^{-1}$. The output LLR $L_{e, D e m}$ is then deinterleaved in $\Pi^{-1}$ and is further decoded by decoder in $C_{1}^{-1}$. The iteration is performed between the demapper and the decoder (ACC is included) until the iteration is considered enough. Finally, a hard decision is made from the aposteriori LLR at the output of Decoder $C_{1}^{-1}$. A detailed Tanner graph between the decoder + doped ACC and the demapper is shown in Fig. 2, where the doping rate $P=2$ is illustrated as an example.

1) Repetition Codes: Repetition codes is one of the simplest errors in correction codes. The principle is only by repeating the information bits for $N$ times with the expectation of error that might occur in the channel and is only affecting a little part of the repetition. Coding rate $R$ of repetition codes is defined as

$$
R=\frac{1}{N} .
$$

Generator and parity check matrices of the Repetition 


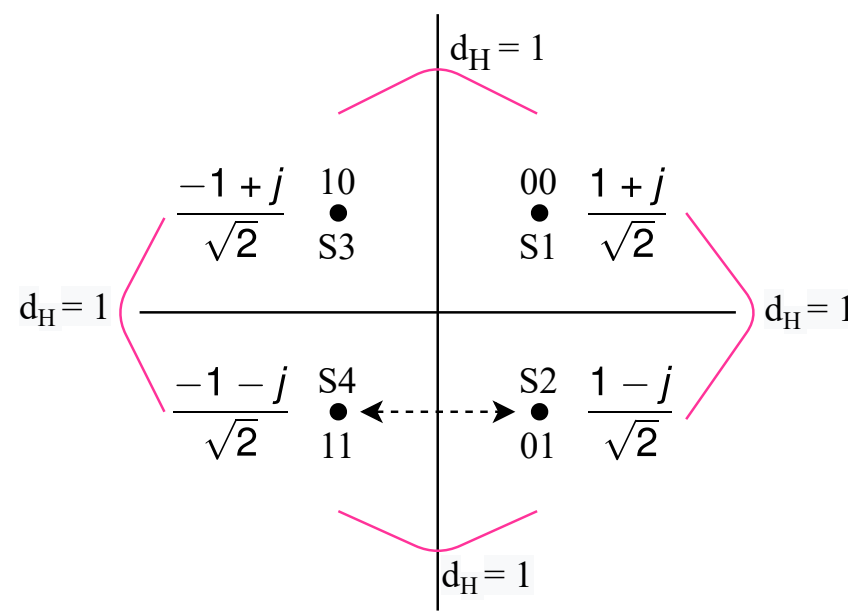

(a)

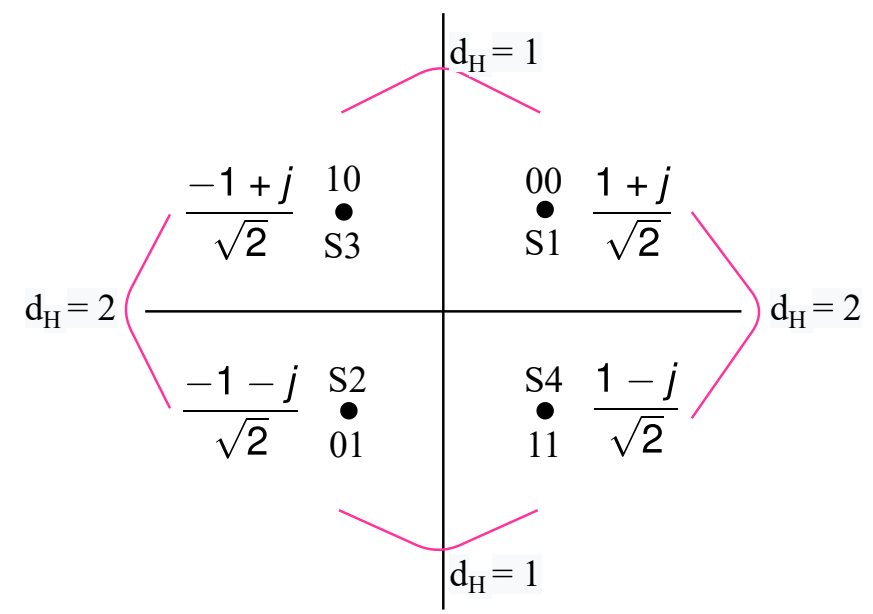

(b)

Fig. 3 Difference in QPSK symbols labelling between (a) Gray mapping and (b) non-Gray mapping.

codes are expressed, respectively, as

$$
\begin{aligned}
\mathbf{G} & =\left[\begin{array}{cccc}
1 & 1 & \cdots & 1
\end{array}\right] \\
\mathbf{H} & =\left[\begin{array}{ccccc}
1 & 1 & 0 & \cdots & 0 \\
1 & 0 & 1 & \cdots & 0 \\
\vdots & \vdots & \vdots & \ddots & \vdots \\
1 & 0 & 0 & \cdots & 1
\end{array}\right] .
\end{aligned}
$$

Therefore, the encoded bits $\mathbf{C}$ in Fig. 1 is expressed as

$$
\mathbf{C}=\mathbf{U} \cdot \mathbf{G}
$$

with $\mathbf{U}$ being the uncoded bits. The estimate bits $\hat{\mathbf{U}}$ is then obtained from the sum-product algorithm of the loglikelihood ratio (LLR) and parity check matrix $\mathbf{H}$.

In this paper, we use repetition codes with $R=1 / 3$, which means that the 1 bit of information is repeated and becomes 3 codewords and $R=1 / 9$, which means that the 1 bit of information is in 9 codewords.

2) Doped Accumulator (ACC): Doped Accumulator is the two-state systematic recursive convolutional (SRC) codes with a coding rate of $R=1 / 2$. Using that coding rate, every 1 bit input $u_{(k)}$ will have 2 bits output, which are $C_{u(k)}$ and $C_{a c c(k)} . C_{u(k)}$ is an interleaved-bit from output of repetition codes, while $C_{a c c(k)}$ is the result of xor operation between $C_{u(k)}$ and $C_{a c c(k-1)}$. To keep $R=1$, bit of $C_{u}(k)$ is replaced by $C_{a c c(k)}$ at every $k=i P$, expressed as [10]

$$
u(k)= \begin{cases}C_{a c c}(k), & k=i P \\ C_{u}(k), & \text { otherwise }\end{cases}
$$

with $i=1,2, \cdots, K / P$, where $K$ is the block length of $C_{a}$ and $P$ is the doping rate.

In this paper, we use $P=2$, which means the second bit and its multiple are replaced by $C_{a c c(k)}$. The decoding from doped accumulator uses Bahl, Cocke, Jelinek dan Raviv (BCJR). Every $k=i P$, BCJR will restore $C_{a c c(k)}$ bit into $C_{u(k)}$ bit when iterative decoding occurs using LLR feedback from the repetition decoder.

This paper uses doped accumulator which is expected to decrease the value of BER on a system. It can also match EXIT chart between demapper and decoder to reach it shortest range without intersecting. Another function of ACC is to increase the spectrum efficiency due to single parity check code (SPC) not being required when ACC is applied [10].

3) Gray and Non-Gray Modulation: This paper modifies (a) Gray QPSK into (b) non-Gray QPSK by swapping the positions of $\mathrm{S} 4$ and $\mathrm{S} 2$ in the constellation diagram showed in Fig. 3. The difference between original mapping or Gray mapping and non-Gray mapping is the Hamming distance and Euclidean distance. The Hamming distance is the number of bits that is not equal between 2 compared symbols. Gray mapping has 1 Hamming distance in every constellation, while non-Gray mapping has 2 Hamming distances between S1-S4 and S2-S3. The Euclidean distance is the distance between 2 compared symbols. The equation to find out the Euclidean distance is

$$
d_{E}=\sqrt{\sum_{i=1}^{n}\left(a_{i}-b_{i}\right)^{2}}
$$

with $n$ being the dimension of constellation point. This non-Gray mapping is designed to make symbols with small Euclidean distance to have a large Hamming distance.

\section{B. Proposed Channel Model}

This paper proposes channel model to represent real condition according to harbor environment characteristic in Indonesia. This model is important for designing an optimal of harbor wireless communication system. If we can identify the appropriate channel model, we can also determine other parameters to increase system performance.

1) New York University Simulator (NYUSIM): NYUSIM is a MATLAB based channel simulator which is developed by New York University (NYU) researcher for modeling channels based on input parameters. NYUSIM is designed for broadband wireless communication system on 
TABLE I

USED PARAMETERS OF HARBOR ENVIRONMENT TO DERIVE THE CHANNEL MODEL FOR HARBOR COMMUNICATIONS

\begin{tabular}{ll}
\hline Parameters & Value \\
\hline Frequency & $3.3 \mathrm{GHz}$ \\
Bandwidth & $20 \mathrm{MHz}$ \\
Scenario & Urban Micro \\
Environment & NLOS \\
TX Power & $30 \mathrm{dBm}$ \\
Barometic Pressure & $1010.5 \mathrm{mbar}$ \\
Humidity & $57 \%$ \\
Temperature & $32^{\circ} \mathrm{C}$ \\
Rain Rate & $120.4 \mathrm{~mm} / \mathrm{hr}$ \\
Foliage Loss & $\mathrm{No}$ \\
RX Location & 1000 \\
\hline
\end{tabular}

millimeter-wave (mmWave) frequency using 3D Statistical Spatial Channel Model (SSCM) [12]. Table. I shows the input parameters for harbor channel model in Indonesia that is represented by Tanjung Priok Harbor, North of Jakarta.

2) Power Delay Profile (PDP): PDP is a distribution value of the received power signal passing the multipath channel. PDP value indicates the dispersion or distribution transmitted on every path [13]. On the PDP plot, power signal on every path is plotted to delay propagation. The output of NYUSIM software called instantaneous PDP, which is a momentarily path value and still fluctuates by time. Instantaneous PDP needs to be changed to a representative PDP. Several steps to get a representative PDP based on [7] are:

1) Determine the harbor environment parameter for NYUSIM input. For bandwidth parameter, use $B_{\text {sim }}=2 B_{\text {actual }}$.

2) Generate instantaneous PDP $i$ from output NYUSIM with $i$ being the index of PDP with the value of $i=$ $1,2, \cdots, L$ (with $L$ as the total instantaneous PDP)

3) Round the delay of every PDP according to Nyquist Theorem that $\Delta T$ rounding range needs to comply with $\Delta T \leq \frac{1}{B_{\text {actual }}}$.

4) Sum all paths on every time index $t$.

5) Take the value of 90th percentile from each CDF path that is used as a representative PDP.

6) Give a threshold of $-140 \mathrm{dBm}$ for every representative PDP, where $-140 \mathrm{dBm}$ is expected as the device sensitivity. All component below $-140 \mathrm{dBm}$ are considered as a noise. ${ }^{1}$

7) Normalize every value of representative PDP.

\section{EXIT Analysis}

This paper uses EXIT chart to visualize the exchange of extrinsic mutual information between demapper and decoder [14]. EXIT chart is pioneered by Stephan ten Brink in [15]. We use Gaussian and fading assumption with J-function $J(\cdot)$

\footnotetext{
${ }^{1}$ This number is chosen by assuming that the device receiver sensitivity in the future is about $-140 \mathrm{dBm}$.
}

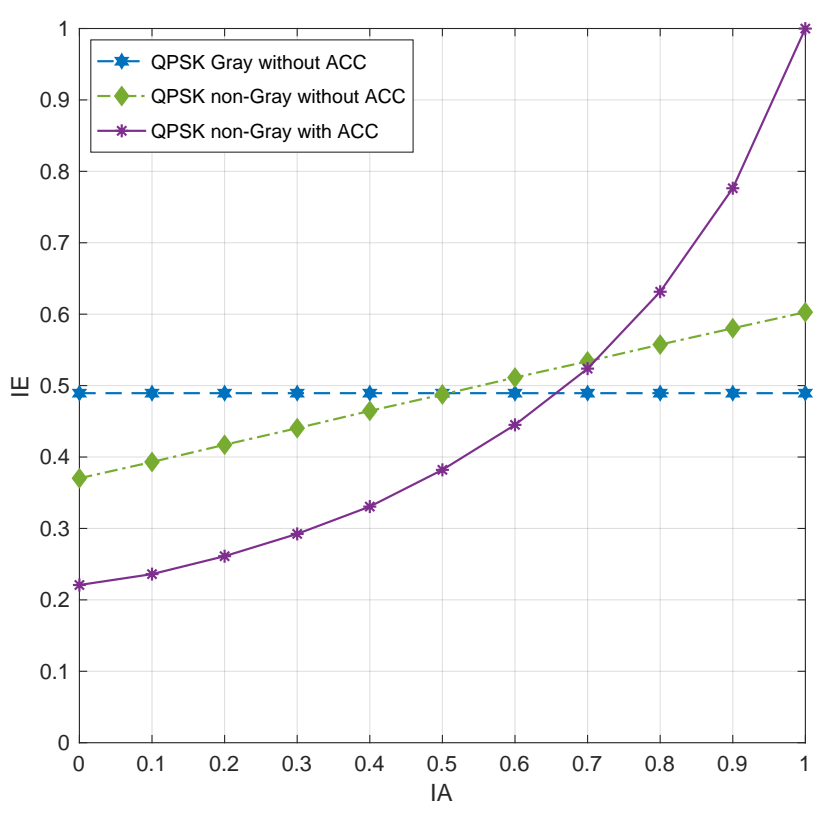

Fig. 4 EXIT chart characteristics of Gray, non-Gray and non-Gray modulations with doped ACC.

to measure mutual information I for modelling exchange extrinsic mutual information. The function is a transformation between variance $\sigma$ using inverse J-functions $J(\cdot)^{-1}$, which is defined as [16]

$$
\sigma=J^{-1}(I)
$$

and

$$
I=J(\sigma)
$$

1) EXIT Chart Demapper: EXIT chart demapper is obtained by $L_{e}$ and $L_{a}$. Extrinsic LLR of the $v-t h$ bit of the demapper $\left(L_{e, D e m}\right)$ is obtained by [10] with $y$ as received signal, $s$ as output mapper symbol, $h s$ as output symbol from channel, $\sigma$ as noise variance, $L_{a, D e m}$ as the a priori LLR of the demapper (obtained from the decoder) to be calculated by

$$
L_{a, D e m}=\frac{\sigma^{2} \cdot(1-2 \cdot q[v])}{2}+\operatorname{randn}(1, \ell(q \mid[v])) \cdot \sigma,
$$

where randn is a random normal distribution function in MATLAB and $\ell$ is the length of LLR. The $\sigma$ is a noise variance obtained from

$$
\sigma=\frac{-1.0 / 0.3037 \cdot \log \left(1.0-I_{a, D e m}^{1.0 / 1.1064}\right)}{\log (2.0)^{1.0 /(2.0 \cdot 0.8935)}}
$$

where $I_{a, D e m}$ is a priori mutual information of demapper. Extrinsic mutual information of demapper $I_{e, D e m}$ is obtained from the histogram calculation between the value of $L_{e, D e m}$ and the information bits. The similarity between $L_{e, D e m}$ and information bits affects the value of $I_{e, D e m}$. If the value of $I_{e, D e m}$ and information bits are equal, the value of $I_{E}$ closes to 1 .

Fig. 4 shows the different characteristics of EXIT chart 


$$
L_{e, D e m}(q,[V])=\log \frac{P(q[v]=0 \mid y)}{P(q[v]=1 \mid y)}=\log \frac{\sum_{s \in s 0} \exp \left\{-\frac{|y-h x|^{2}}{2 \sigma^{2}}\right\} \prod_{w=1, w \neq v}^{l} \exp \left\{-q[w] L_{a, \operatorname{Dem}(q[w])}\right\}}{\sum_{s \in s 1} \exp \left\{-\frac{|y-h x|^{2}}{2 \sigma^{2}}\right\} \prod_{w=1, w \neq v}^{l} \exp \left\{-q[w] L_{a, \operatorname{Dem}(q[w])}\right\}}
$$

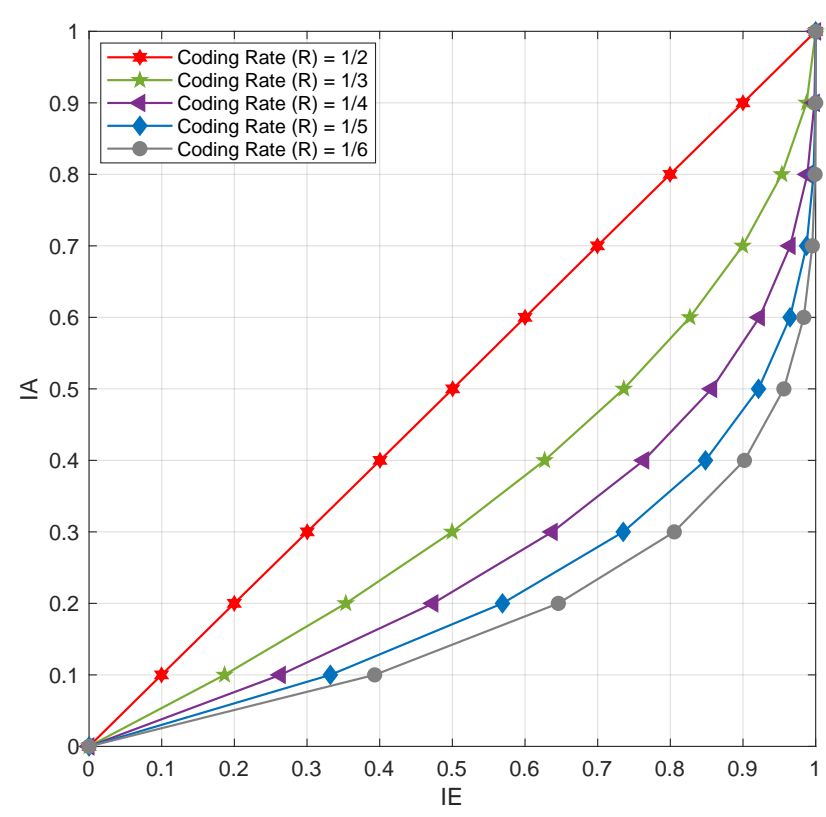

Fig. 5 EXIT chart of the repetition decoder with coding rates of $1 / 2,1 / 3$, $1 / 4,1 / 5$, and $1 / 6$.

demapper between Gray modulation, non-Gray modulation and non-Gray modulation with doped ACC in SNR $\gamma=0 \mathrm{~dB}$. Gray modulation has flat EXIT curve. Therefore, it does not match with EXIT curve repetition decoder. Non-Gray modulation has a sloping EXIT curve, therefore quite suitable with EXIT curve repetition decoder. While Non-Gray modulation with doped ACC has more suitable curve that does not intersect EXIT curve with EXIT curve repetition decoder.

2) EXIT Chart Repetition Decoder: For Repetition decoder, the extrinsic mutual information in the block-by-block decoding is defined as [8].

$$
I_{e, D e c}=J\left(\sqrt{\left(d_{v}-1\right) \cdot J^{-1}\left(I_{a, D e c}\right)^{2}}\right)
$$

with

$$
I_{a, D e c}=I_{e, D e m} .
$$

$I_{e, D e c}$ is an extrinsic mutual information of repetition decoder, $J(\cdot)$ is the function that convert $\sigma$ of LLR to its corresponding mutual information $I(\cdot), J(\cdot)^{-1}$ is the inverse of $J(\cdot), d_{v}$ is the degree of variable nodes, $I_{a, D e c}$ is a priori mutual information of repetition decoder and $I_{e, D e m}$ is an extrinsic mutual information of demapper. Fig. 5 shows an EXIT chart characteristic of repetition decoder with some coding rate. The horizontal axis represents $I E$ and the

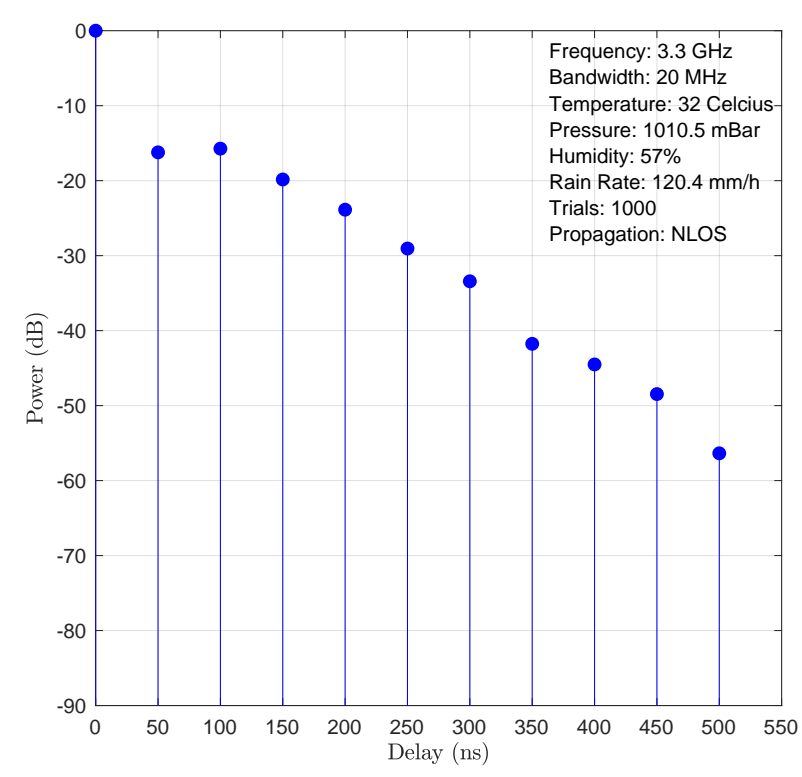

Fig. 6 Representative PDP for the harbor channel model in Indonesia.

vertical axis represents $I A$.

\section{RESUlts AND Discussion}

\section{A. Representative PDP}

Channel model is proposed based on the representative PDP as shown in Fig. 6. The representative PDP in this paper then mapped at every $\triangle T 50 \mathrm{~ns}$ obtained from

$$
\Delta T \leq \frac{1}{B_{\text {actual }}}
$$

with $\triangle T$ being a difference between index delay and $B W_{\text {actual }}$ (applied bandwidth). From the simulation, we obtain the result of 11 path. In this paper, we use $3.3 \mathrm{GHz}$ frequency and $20 \mathrm{MHz}$ bandwidth. Consequently, OFDM numerology 0 is used [17]. Numerology 0 is more suitable if low latency and low power consumption are desired as it has the smallest bandwidth, subcarrier spacing, and FFT size compared to other numerology [4].

For OFDM, the value of path must be less or equal to $\mathrm{CP}$ length to avoid ISI [18]. For OFDM numerology 0, CP length is $Q \approx 10$ according to the

$$
Q=\frac{T_{c p}(\mu)}{T_{O F D M}(\mu)} \cdot N_{F F T},
$$

where $Q$ is CP length, $T_{c p}$ is CP duration, $T_{O F D M}$ is OFDM symbol duration and $N_{F F T}$ is FFT size. This paper uses $N_{F F T}=128, T_{c p}=4,69 \mu \mathrm{s}$ and $T_{O F D M}=66,67 \mu \mathrm{s}$ according to OFDM numerology 0 classification. As the 


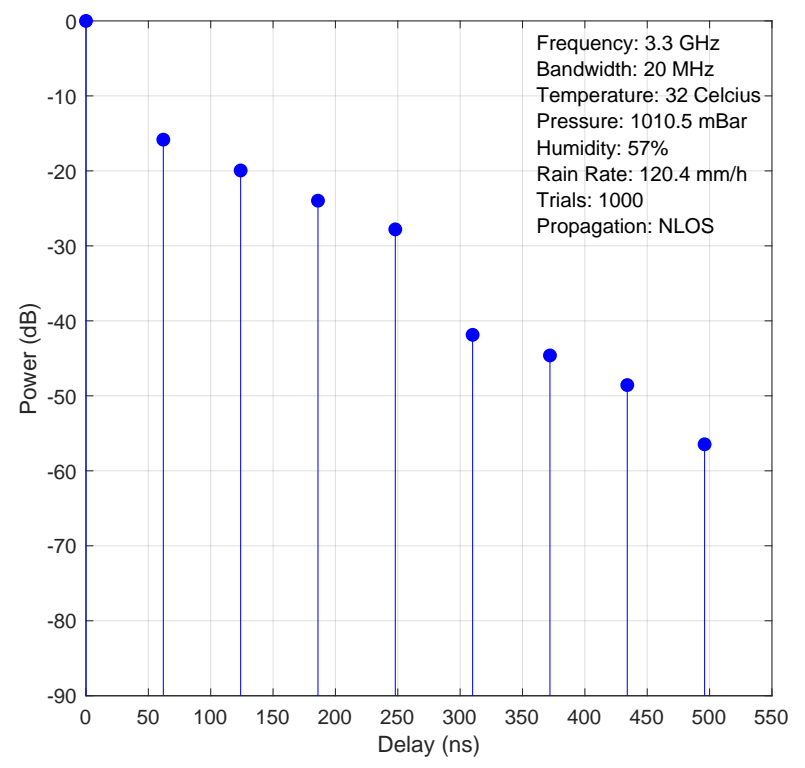

Fig. 7 The proposed harbor channel model of Indonesia.

number of paths is more than $Q$, we need to do a scaling method with interval 62 ns to decrease the number of paths. The result of representative PDP is mapped according to the scaling method shown in Fig. 7.

\section{B. Outage Performance}

Outage performance is the chance of an outage that causes information to be not be decoded. Outage performance is obtained from outage probability calculation, which is the probability of channel capacity $C$ falling below the channel coding rate $R$ that can be defined as

$$
P_{\text {out }}=P(R>C) \text {. }
$$

The compared channel coding rate are $R=\{1,1 / 3,1 / 9\}$, while channel capacity $C$ obtained from $\mathrm{CDF}$ evaluation is calculated from channel coding rate $R=\{1,1 / 3,1 / 9\}$. While the value of channel capacity $C$ can be calculated by

$$
C=\frac{B}{N} \cdot \sum_{n=1}^{N} \log _{2}\left(1+\left|\psi_{n}\right|^{2} \cdot \gamma\right),
$$

where $B$ is bandwidth, $\psi$ is eigenvalue defined as

$$
\psi=\operatorname{diag}\left(\mathbf{F} \mathbf{H}_{c} \mathbf{F}^{H}\right),
$$

and $\gamma$ is signal to noise ratio (SNR), where $F$ is Fast Fourier Transform (FFT) matrix, $\mathbf{H}_{c}$ is channel matrix after CP removal, and $F^{H}$ is Inverse Fast Fourier transform (IFFT) matrix [5].

Fig. 8 shows an outage performance from the simulation. The simulation uses QPSK modulation with FFT size 128 and 500.000 channel realization. From the figure, multipath channel with harbor path has a better performance than the single path channel caused by diversity effect. Usage of channel coding rate also impacted the obtained outage

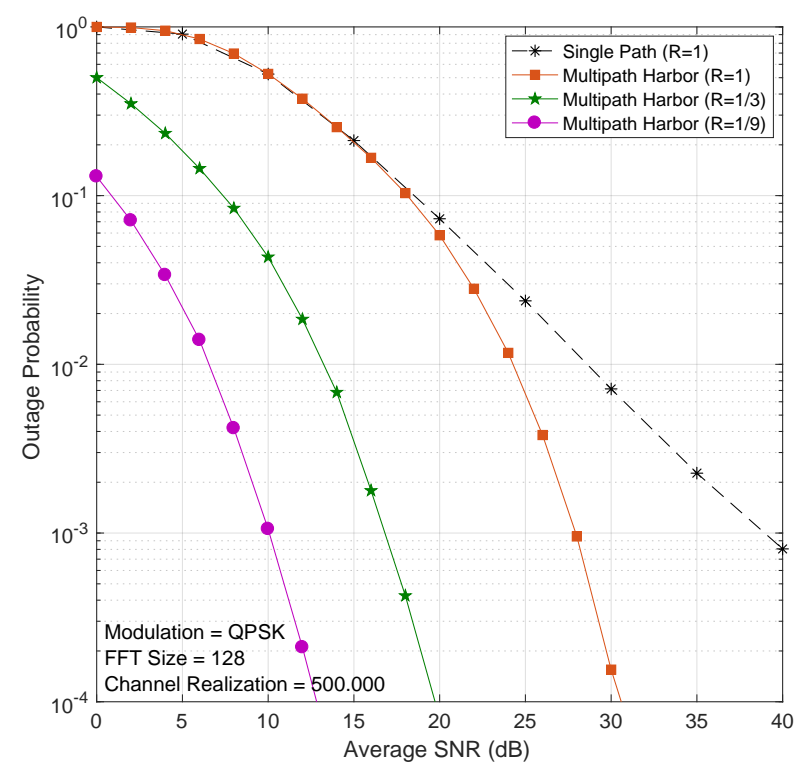

Fig. 8 Outage performance of harbor channel model.

performance curve. On harbor channel with $R=1$, outage probability $10^{-3}$ is obtained on SNR $27.9 \mathrm{~dB}$, on harbor channel model with $R=1 / 3$, outage probability $10^{-3}$ is obtained on SNR $16.7 \mathrm{~dB}$, while on harbor channel model with $R=1 / 9$, outage probability $10^{-3}$ is obtained on SNR $10 \mathrm{~dB}$. Hence, it can be concluded that outage curve with $R=1 / 9$ has better performance. Outage performance might demonstrate the channel quality in theory, as it is derived from Shannon limit. Therefore, it can be a reference for FER testing and hardware implementation testing on Indonesian harbor.

\section{Optimal Soft Demapper Performance}

This paper performs a simulation using MATLAB software, where a maximum limit of 710 and minimum of 710 for LLR calculations is of interest. Due to this limit, the demapper must be designed to handle an infinite value that might result in an error floor at a certain SNR. The demapper limit for Gray mapping has been proposed in [19], while for non-Gray mapping, the demapper limit has not been proposed. Therefore, this paper proposes the best demapper limit for simulation on QPSK modulation for nonGray mapping using the same method as [19].

This paper observes $d_{P}$ from 0.001 to 1000 at SNR $22 \mathrm{~dB}$ and $30 \mathrm{~dB}$ on Rayleigh fading channels in non-Gray QPSK modulation using the repetition codes and doped ACC shown in Fig. 9. The figure shows the difference average BER values for each $d_{P}$. We select $d_{P}$ to have minimum BER. At SNR $22 \mathrm{~dB}$ and $30 \mathrm{~dB}$, the best BER performance is obtained when $d_{P}=1$, therefore we use $d_{P}=1$ as the demapper limit. 


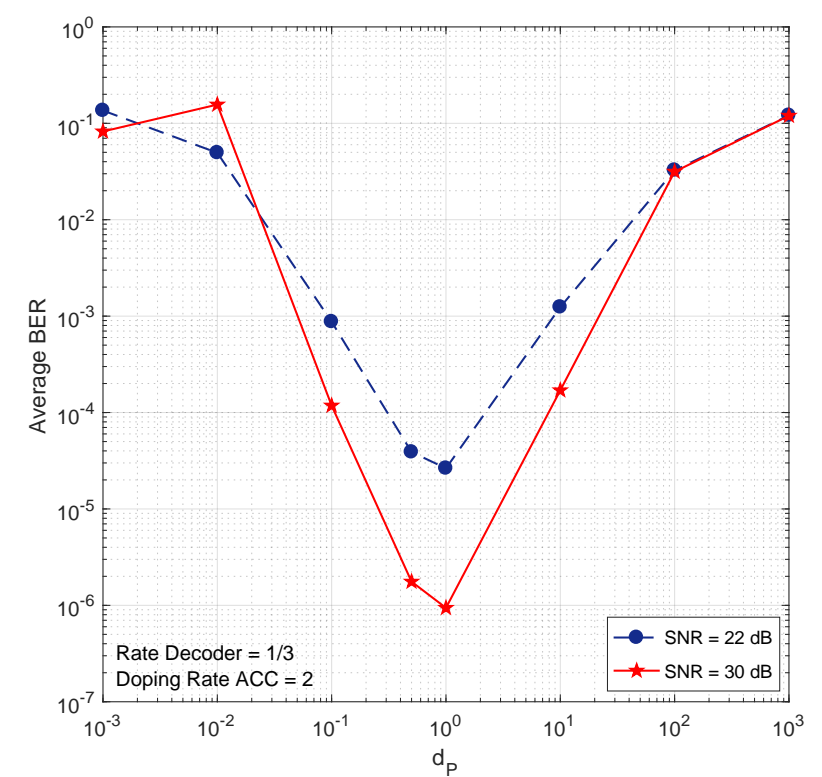

Fig. 9 The BER performances of non-Gray QPSK modulation with repetition codes plus dopped ACC in multipath fading channels.

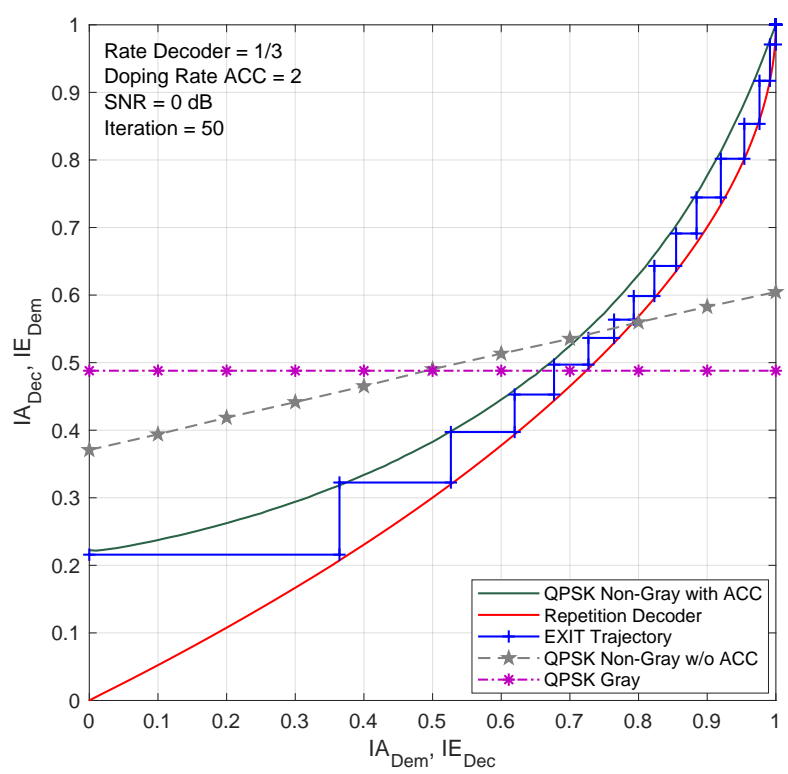

Fig. 10 Optimal EXIT chart between non-Gray QPSK demapper with ACC and repetition decoder on AWGN channels.

\section{EXIT Chart}

EXIT chart is used to predict the convergence of the iterative decoding process [20]. The EXIT chart is also used to match the EXIT curve demapper and decoder to find the smallest gap. This paper evaluates EXIT chart on AWGN and fading channels with path derived from the proposed harbor channel model.

1) EXIT chart on AWGN channel: Fig. 10 shows optimal EXIT chart between demapper and repetition decoder on

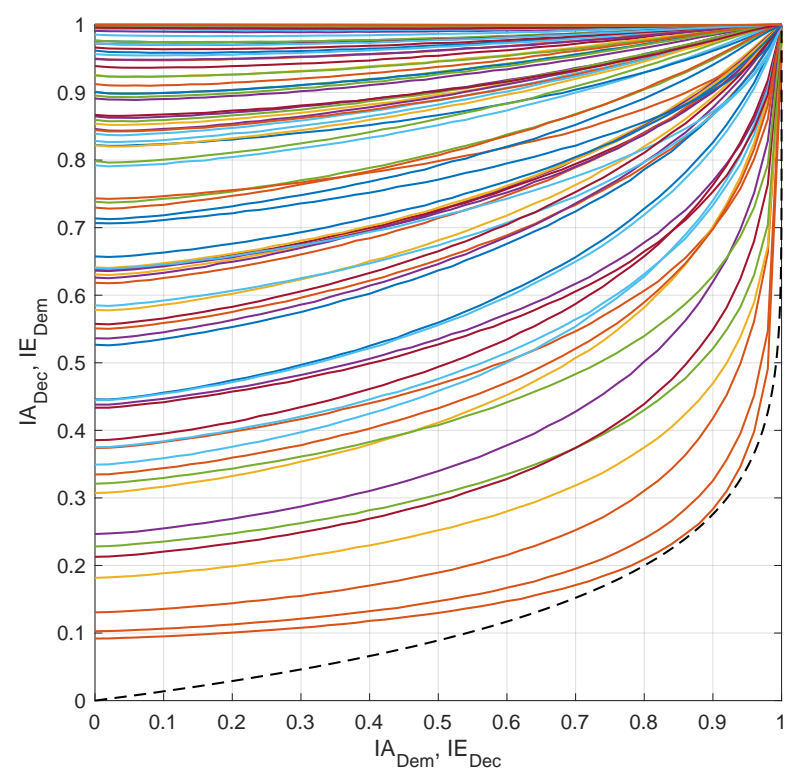

Fig. 11 Optimal EXIT chart between non-Gray QPSK demapper with ACC and repetition decoder on fading channels.

AWGN channel. The $\mathrm{x}$-axis shows $I A$ on the EXIT curve demapper and $I E$ on the EXIT curve decoder, while the yaxis shows $I A$ on the EXIT curve decoder and $I E$ on the EXIT curve demaapper. The closest gap is obtained between EXIT curve repetition decoder with a rate of $R=1 / 3$ and EXIT curve non-Gray modulation with doped ACC with dopping rate $P=2$. The simulation is done by using 50 iterations with SNR $0 \mathrm{~dB}$. This EXIT chart is also accompanied by EXIT trajectories, which is represented by the zig-zag line. EXIT trajectory is plotted based on histogram calculations between LLR and information bits. EXIT trajectories shows actual behavior of iterative decoding [16]. From Fig. 10 shows that the EXIT chart and the trajectory match each other indicating that the EXIT chart is correct..

2) EXIT chart on fading channel: Fig. 11 shows optimal EXIT chart between demapper and repetition decoder on fading channel. The x-axis shows $I A$ on the EXIT curve demapper and $I E$ on the EXIT curve decoder, while the y-axis shows $I A$ on the EXIT curve decoder and $I E$ on the EXIT curve demapper. The solid line shows the EXIT curve demapper with 100 different channels. Channels in fading channels are random and thus produce a different EXIT curve, while the dotted line shows the EXIT curve repetition decoder. Closest gap is obtained between EXIT curve repetition decoder with rate $R=1 / 9$ and EXIT curve non-Gray modulation with doped ACC with dopping rate $P=2$. The simulation is done using 50 iterations with SNR $10 \mathrm{~dB}$.

\section{E. BER Performances}

BER performances are evaluated under AWGN and fading channels with QPSK modulation. BER theory of AWGN 


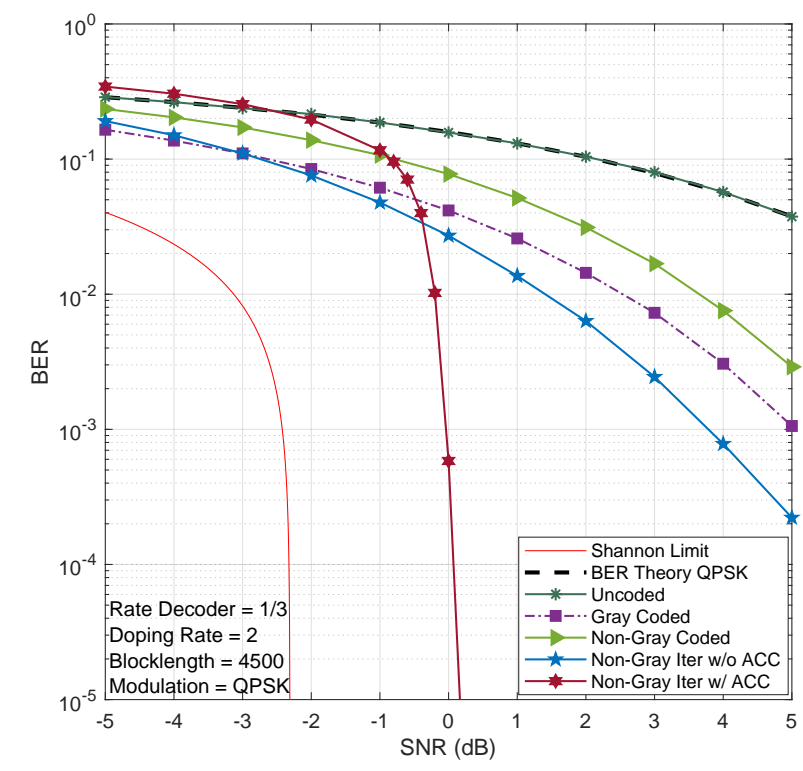

Fig. 12 Comparison of BER performance between Gray and non-Gray modulation with and without iterative decoding and as well as with doped accumulator with a doping rate of $P=2$ and a coding rate of $R=1 / 3$ in AWGN channel.

channel based on [21] is

$$
P_{b, A W G N}=\frac{1}{2} \operatorname{erfc}\left(\sqrt{\frac{E_{b}}{N_{0}}}\right),
$$

while BER theory for fading channel is

$$
P_{b, \text { Fading }}=\frac{1}{2}\left[1-\frac{1}{\sqrt{1+\frac{1}{E_{b} / N_{0}}}}\right]
$$

with $E_{b} / N_{0}$ being energy per bit to noise power spectral density ratio. Relationship between $S N R$ and $E_{b} / N_{0}$ is

$$
\gamma=\frac{E_{b}}{N_{0}} \cdot R \cdot M
$$

where $\gamma$ is the SNR, $R$ is the channel coding rate and $M$ is the modulation index.

1) BER Performance in AWGN Channel: BER performance in AWGN channel is shown in Fig. 12 used to verify the EXIT chart of proposed channel coding. The simulation is performed using 9000 information bits. The channel coding rate used is $R=1 / 3$ and the doping rate accumulator is $P=2$. The value was chosen as it is the most suitable combination based on the results of the EXIT chart. Horizontal axis is represented as SNR and vertical axis is represented as BER.

BER theory and uncoded have the same performance. The efficiency of SNR can be increased by using channel coding. QPSK Gray modulation have BER performances $10^{-3}$ achieved at SNR $\gamma=5 \mathrm{~dB}$. QPSK non-Gray modulation have BER performances $10^{-3}$ achieved at SNR $\gamma=5.9 \mathrm{~dB}$. QPSK non-Gray modulation with iterative decoding have

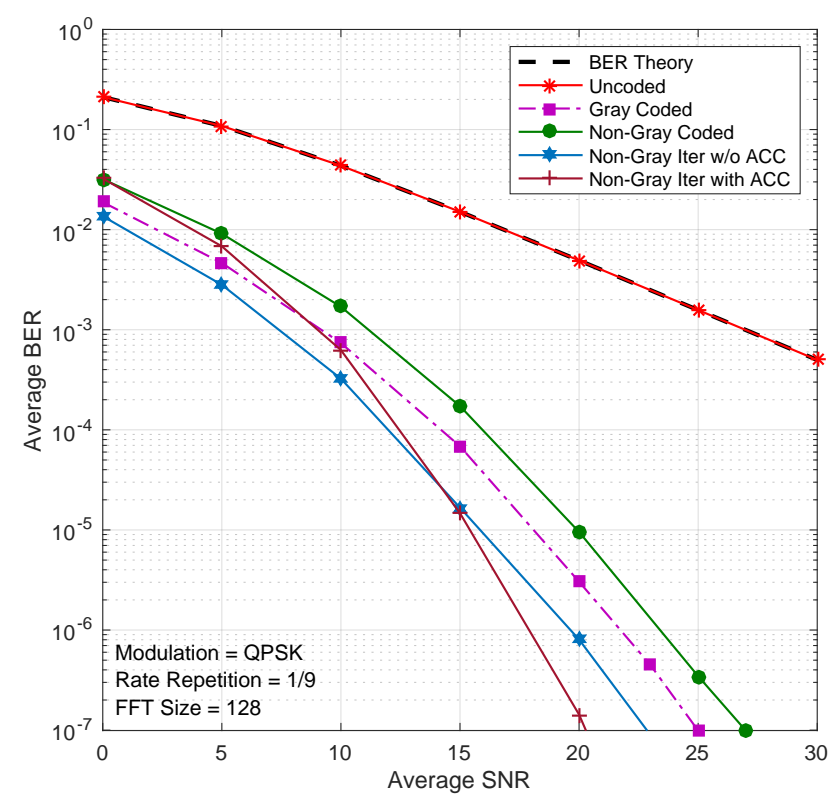

Fig. 13 Comparison of BER performance between Gray and non-Gray modulation with and without iterative decoding as well as with doped accumulator with a doping rate of $P=2$ and a coding rate of $R=1 / 9$ in fading channel.

BER performances $10^{-3}$ achieved at SNR $\gamma=3.8 \mathrm{~dB}$. QPSK non-Gray modulation plus accumulator with iterative decoding have BER performances $10^{-3}$ achieved at SNR $\gamma=0 \mathrm{~dB}$. Therefore, the proposed channel coding with doping rate $P=2$ has a distance of $2.18 \mathrm{~dB}$ from the Shannon limit.

2) BER Performance in Fading Channel: BER performance used for the proposed channel coding performance analysis on harbor channel model is shown in Fig. 13. The path is used from proposed harbor channel model represented by 9 paths. The system uses OFDM with numerology 0 and QPSK Gray and non-Gray modulation. The simulation uses 128 FFT size with 100000 channels realization. The repetition rate used is $1 / 9$ and dopping rate used is 2 . Horizontal axis is represented as average SNR and vertical axis is represented as average BER.

BER theory and uncoded have the same performance. The efficiency of SNR can be increased by using channel coding. QPSK Gray modulation have BER performances $10^{-6}$ achieved at SNR $\gamma=21.5 \mathrm{~dB}$. QPSK non-Gray modulation have BER performances $10^{-6}$ achieved at SNR $\gamma=23.1 \mathrm{~dB}$. QPSK non-Gray modulation with iterative decoding have BER performances $10^{-6}$ achieved at SNR $\gamma=20.1 \mathrm{~dB}$. QPSK non-Gray modulation plus accumulator with iterative decoding have BER performances $10^{-6}$ achieved at SNR $\gamma=17.6 \mathrm{~dB}$. Therefore, the proposed channel coding with doping rate $P=2$ able to improve system performance. 


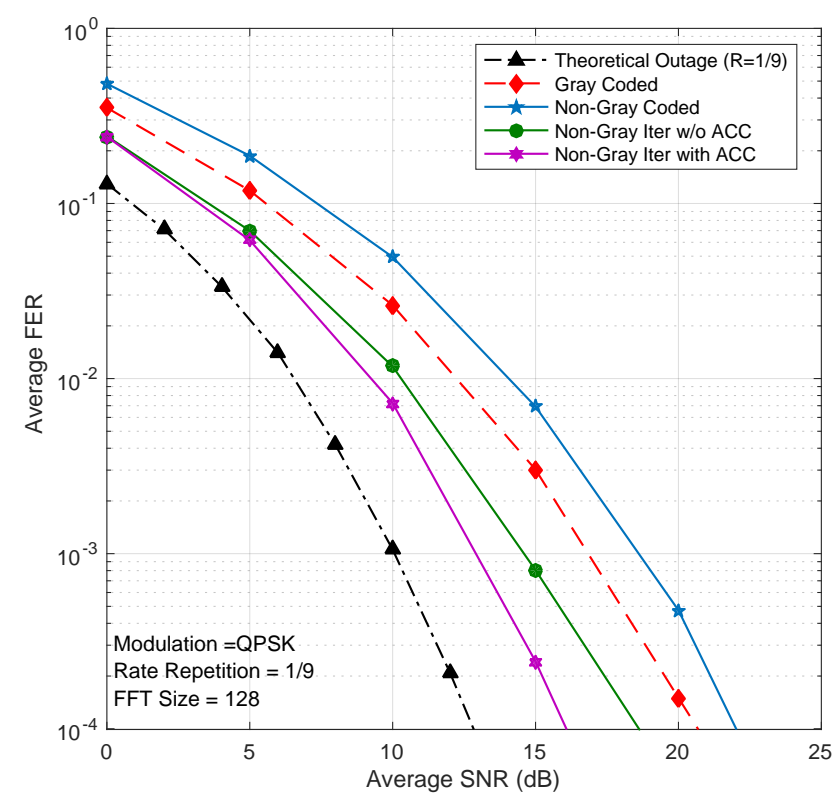

Fig. 14 Comparison of FER performance between Gray and non-Gray modulation with and without iterative decoding as well as with doped accumulator with a doping rate of $P=2$ and a coding rate of $R=1 / 9$ in fading channel.

\section{F. FER Performances}

Outage performances are validated using FER performance curve. FER and outage performance must have the same gradient, since they use the same channel coding. Fig. 14 presents FER performances in multipath fading channel with a path from harbor channel model that uses the OFDM and QPSK modulation. The simulation uses 128 FFT size, with a channel coding rate of $1 / 9$ with a doping rate accumulator of 2. Horizontal axis is represented as average SNR and vertical axis is represented as average FER.

Outage performance and uncoded have the same gradient. The efficiency of SNR can be increased by using channel coding. QPSK Gray modulation have FER performances $10^{-3}$ achieved at SNR $\gamma=17 \mathrm{~dB}$. QPSK non-Gray modulation have FER performances $10^{-3}$ achieved at SNR $\gamma=18.6 \mathrm{~dB}$. QPSK non-Gray modulation with iterative decoding have FER performances $10^{-3}$ achieved at SNR $\gamma=14.5 \mathrm{~dB}$. QPSK non-Gray modulation plus accumulator with iterative decoding have FER performances $10^{-3}$ achieved at SNR $\gamma=12.9 \mathrm{~dB}$. Therefore, the proposed channel coding with doping rate $P=2$ able to improve system performance.

\section{CONCLUSiON}

This paper has proposed (i) harbor channel model and (ii) simple iterative repetition decoding for harbor wireless communications. The proposed channel model is obtained from computer simulations using the real-field environmental parameters of Tanjung Priok harbor. The proposed harbor channel model has 9 paths, of which the FER performances has been validated using the theoretical outage performances. The repetition codes have been proposed and designed from the simplest channel coding to be one of strong channel coding schemes after being combined with a non-Gray QPSK demapper helped by an ACC. The accumulator EXIT curve is controlled using a doping rate, which is optimized using EXIT analysis.

We found that a doping rate $P=2$ and coding rate $R=1 / 3$ are good since this design provides a BER cliff approaching the Shannon limit with a distance of only about $2 \mathrm{~dB}$ on the AWGN channel. The similar approach was used to optimize the coding rate and doping rate for harbor channel model, where we found that the EXIT curve between non-Gray QPSK with accumulator and a doping rate $P=2$ and repetition decoder $R=1 / 9$ has small gaps for almost all channel realizations providing excellent performance although in multipath fading harbor channels. The results of this paper are expected to reduce the power consumption of logistic monitoring devices at the harbor without reducing the quality of communications.

\section{REFERENCES}

[1] P. P. I. II, “Annual report 2018 membangun pelabuhan digital yang unggul," PT Pelabuhan Indonesia II (Persero), Tech. Rep., 2018.

[2] H. Laut. (2019) Industri pelabuhan dan pelayaran bersiap hadapi revolusi 4.0. [Online]. Available: http://hubla.dephub.go.id/berita/ Pages/IndustriPelabuhandanPelayaranBersiapHadapiRevolusi4.0.aspx

[3] J. Liu, "Wireless multipath fading channels modeling and simulation based on sum-of sinusoids," in 2016 First IEEE International Conference on Computer Communication and the Internet, Changsha, China, 2016, pp. 165-168.

[4] E. Christy, R. P. Astuti, and K. Anwar, "Telkom university 5G channel models under foliage effect and their performance evaluations," in 2018 International Conference on ICT for Rural Development (ICICT RuDev), 2018, pp. 29-34.

[5] R. D. Wahyuningrum, K. Anwar, and L. O. Nur, "Humidity effect to the indonesia $5 \mathrm{G}$ channel model at $3.3 \mathrm{GHz}$," in 2019 Symposium on Future Telecommunication Technologies (SOFTT), vol. 1, 2019, pp. $1-5$.

[6] M. Alfaroby, K. Anwar, and N. M. Ardiansyah, "5G channel model indonesia menggunakan teknik statistical spatial channel model (SSCM) indonesia $5 \mathrm{G}$ channel model based on statistical spatial channel model (SSCM)," in e-Proceeding of Engineering, Indonesia, Maret 2016, pp. $107-115$.

[7] M. N. Rahman, K. Anwar, and L. O. Nur, "Indonesia 5G channel model considering temperature effects at $28 \mathrm{GHz}$," in 2019 Symposium on Future Telecommunication Technologies (SOFTT), vol. 1, 2019, pp. 1-6.

[8] D. Zhao, A. Dauch, and T. Matsumoto, "Modulation doping for repetition coded BICM-ID with irregular degree allocation," in 2009 International ITG Workshop on Smart Antennas, February 2009, pp. 312-318.

[9] K. Fukawa, D. Zhao, A. Tolli, and T. Matsumoto, "Irregular repetition and single parity check coded BICM-ID using extended mapping -optimal node degree allocation-," in 2010 5th International ICST Conference on Communications and Networking in China, 2010, pp. 1-6.

[10] K. Anwar and T. Matsumoto, "Very simple BICM-ID using repetition code and extended mapping with doped accumulator," in Wireless Pers Commun 67, September 2011, p. 573-584.

[11] Y. Romadhon, "Optimalisasi pelabunah tanjung priok menuju pelabuhan berkelas dunia,” Jurnal Logistik Indonesia, 2018.

[12] S. Sun, G. R. MacCartney, and T. S. Rappaport, "A novel millimeterwave channel simulator and applications for $5 \mathrm{G}$ wireless communications," in 2017 IEEE International Conference on Communications (ICC), May 2017, pp. 1-7. 
[13] R. SAHA, "Power delay profile and channel classification in cellular mobile communications/ a handbook on cellular mobile communication laboratory a matlab-based approach,” KDDI Research, Tech. Rep., 2016.

[14] S. Brink, "Designing iterative decoding schemes with the extrinsic information chart," AEU-Archiv fur Elektronik und Ubertragungstechnik, vol. 54, 012000.

[15] J. Hagenauer, "The EXIT chart - introduction to extrinsic information transfer in iterative processing," in 2004 12th European Signal Processing Conference, 2004, pp. 1541-1548.

[16] S. ten Brink, "Convergence behavior of iteratively decoded parallel concatenated codes," IEEE Transactions on Communications, vol. 49, no. 10, pp. 1727-1737, 2001.

[17] J. Vihriälä, A. A. Zaidi, V. Venkatasubramanian, N. He, E. Tiirola, J. Medbo, E. Lähetkangas, K. Werner, K. Pajukoski, A. Cedergren, and R. Baldemair, "Numerology and frame structure for $5 \mathrm{G}$ radio access," in 2016 IEEE 27th Annual International Symposium on Personal, Indoor, and Mobile Radio Communications (PIMRC), 2016, pp. $1-5$.

[18] H. Nourollahi and S. Maghrebi, "Evaluation of cyclic prefix length in OFDM system based for rayleigh fading channels under different modulation schemes," in 2017 IEEE Symposium on Computers and Communications (ISCC). Los Alamitos, CA, USA: IEEE Computer Society, jul 2017, pp. 164-169. [Online]. Available: https://doi.ieeecomputersociety.org/10.1109/ISCC.2017.8024523

[19] A. Syukra, K. Anwar, and D. M. Saputri, "On the design of optimal soft demapper for $5 \mathrm{G} \mathrm{nr}$ wireless communication systems," in The 10th Electrical Power, Electronics, Communications, Controls and Inforatics Seminar 2020, 2020.

[20] A. Ashikhmin, G. Kramer, and S. Brink, "Extrinsic information transfer functions: Model and erasure channel properties," Information Theory, IEEE Transactions on, vol. 50, pp. 2657 - 2673, 122004.

[21] H. Harada and R. Prasad, Simuation and Software Radio for Mobile Communiction. Artech House, Inc.685 Canton St. Norwood, MA United States, 2002, ch. 3. 